\title{
SCIENTIFIC REPRTS OPEN Toxicity of diatom-derived polyunsaturated aldehyde mixtures on sea urchin Paracentrotus lividus development
}

Received: 16 March 2018

Accepted: 3 December 2018

Published online: 24 January 2019

\author{
Nadia Ruocco ${ }^{1,2,3}$, Concetta Annunziata ${ }^{1}$, Adrianna lanora ${ }^{1}$, Giovanni Libralato ${ }^{2}{ }^{2}$, \\ Loredana Manfra ${ }^{1,4}$, Susan Costantini ${ }^{5}$ \& Maria Costantini ${ }^{1}{ }^{1}$
}

Diatom-derived polyunsaturated aldehydes (PUAs), decadienal, heptadienal and octadienal, derive from the oxidation of fatty acids and have cytotoxic and anticancer effects. PUAs, tested separately, induce malformations in sea urchin Paracentrotus lividus embryos. Decadienal induces the worst malformations and lowest survival rates. Interestingly, decadienal, heptadienal and octadienal place in motion several genes to counteract their negative effects. To date, no studies are available reporting on the effects of PUA mixtures on marine invertebrates. Here we test binary and ternary mixtures on embryonic development of $P$. lividus. Our findings demonstrate that mixtures of PUAs act (i) at morphological level in synergistic way, being much more severe compared to individual PUAs; (ii) at molecular level also reveal an additive effect, affecting almost all fifty genes, previously tested using individual PUAs. This study is relevant from an ecological point of view since diatoms are a major food source for both pelagic and benthic organisms. This work opens new perspectives for understanding the molecular mechanisms that marine organisms use in reacting to environmental natural toxin mixtures such as diatom PUAs.

Marine organisms are constantly subjected to a mixture of environmental stressors and natural and/or dissolved anthropogenic compounds, including both physical and chemical ${ }^{1}$. In the last two decades several studies have been performed on chemical toxicants to address scientific questions in mixture toxicology ${ }^{2-6}$. Environmental policy has recognized in mixture effects the major issue in environmental risk assessment ${ }^{2}$. In a situation of exposure to multiple xenobiotics, single compounds may act independently as in a single exposure, or they may interact to modulate the effects of total multiple exposure ${ }^{7}$. The interactions of components in a mixture can result in synergistic, antagonistic or additive toxicity ${ }^{8,9}$.

Among natural toxins, representing a major source of stress for marine organisms, many studies have been performed on diatom-derived secondary metabolites known as oxylipins. These natural compounds are the end-products of a lipoxygenase/hydroperoxide lyase metabolic pathway, triggered by cell damage or breakage, as during grazing by predators or by lysis of cells at the end of diatom blooms ${ }^{10-15}$. Of the known oxylipins, PUAs are the most comprehensively studied because they were the first group described ${ }^{16}$ and are also commercially available, inexpensive and sufficiently stable to allow for a range of laboratory bioassays to be conducted. For these reasons, it has been extensively demonstrated that PUAs are able to affect the embryonic development of several marine invertebrates such as starfish and polychaetes ${ }^{17-19}$, ascidians ${ }^{20,21}$, copepods (reviewed by Ianora and Miralto $)^{22}$ and sea urchins ${ }^{23-31}$. Moreover, in human cancer cell lines PUAs activated cell death ${ }^{32}$. All these studies have been performed testing the three most common PUAs, decadienal, heptadienal and octadienal, separately. At lower concentrations PUAs (decadienal from 0.5 to $2.5 \mu \mathrm{M}$, heptadienal from 1.0 to $6.0 \mu \mathrm{M}$; octadienal

${ }^{1}$ Department of Marine Biotechnology, Stazione Zoologica Anton Dohrn, Villa Comunale, 80121, Napoli, Italy. ${ }^{2}$ Department of Biology, University of Naples Federico II, Complesso Universitario di Monte Sant'Angelo, Via Cinthia, 80126, Napoli, Italy. ${ }^{3}$ Bio-Organic Chemistry Unit, Institute of Biomolecular Chemistry-CNR, Via Campi Flegrei 34, Pozzuoli, Naples, 80078, Italy. ${ }^{4}$ Institute for Environmental Protection and Research (ISPRA), Rome, Italy. ${ }^{5}$ Cancer Research Center of Mercogliano, Istituto Nazionale Tumori -IRCCS - Fondazione G. Pascale, 80131, Napoli, Italy. Nadia Ruocco and Concetta Annunziata contributed equally. Correspondence and requests for materials should be addressed to M.C. (email: maria.costantini@szn.it) 
from 2.0 to $9.0 \mu \mathrm{M}$ ) induced a dose-dependent increase in the number of malformed embryos in the sea urchin Paracentrotus lividus, with decadienal inducing the strongest effects $(2.0 \mu \mathrm{M})^{29}$. PUAs induced apoptosis in $P$. lividus embryos at higher concentrations (decadienal $3.3 \mu \mathrm{M}$, heptadienal $9.0 \mu \mathrm{M}$ and octadienal $11.0 \mu \mathrm{M})^{27}$.

The potential level of real environmental exposure to the PUAs has been reported in Ribalet et al. ${ }^{33}$. These authors estimated that the release of PUAs from each diatom cell was $46.9,4.7$ and $0.5 \mu \mathrm{mol} \mathrm{PUA} \mathrm{l}^{-1}$ considering a distance of 1,10 and $100 \mu \mathrm{m}$ from the cell surface. These data were well within the significant range for affecting growth and performance of surrounding organisms ${ }^{22}$.

In our studies, we used as model organism to test PUAs mixtures the sea urchin P. lividus, which is a well-established marine model species in eco-toxicological studies, being constantly exposed to environmental toxicants and emerging pollutants ${ }^{34-36}$ and very useful for experimental studies and microscopic observations thanks to its high fecundity and the large number of synchronous and transparent embryos produced ${ }^{37,38}$. In particular, we incubated $P$. lividus sea urchin eggs with binary and ternary mixtures of different PUA concentrations, fertilized and followed embryonic development until pluteus stages at 48 hours post fertilization. In order to identify genes affected by PUA mixtures, we also analyzed the expression levels of fifty genes (previously analyzed using the three PUAs separately), having a key role in a broad range of functional responses, such as development, differentiation, skeletogenesis, stress and detoxification processes. The rationale of this work is based on the consideration that organisms are exposed to oxylipin mixtures during direct feeding on diatoms or due to direct exposure, when these compounds are released in seawater following diatom cell lysis in the marine environment. Our aim was to study the possible toxigenic effects of PUA mixtures on sea urchin P. lividus embryos using morphological and molecular approaches.

\section{Results}

Effects of PUA mixtures on sea urchin development. To define PUA mixture effects on sea urchin embryos we checked the following endpoints in P. lividus reproduction and embryonic development: (i) fertilization success (immediately after the addition of sperm which induces an evident elevation of the fertilization membrane in sea urchin eggs); (ii) first cleavage division (leading to two blastomeres, at about $1 \mathrm{hpf}$ ); (iii) pluteus stage (at $48 \mathrm{hpf}$ ). The aldehyde mixtures tested refer to the concentrations reported in Varrella et al. ${ }^{29}$ which showed that concentrations of $1.6 \mu \mathrm{M}$ decadienal, $3.0 \mu \mathrm{M}$ heptadienal and $4.5 \mu \mathrm{M}$ octadienal tested individually induced the same proportion of malformed embryos (about 35\%). With the three binary PUA mixtures, (decadienal $1.6 \mu \mathrm{M}+$ heptadienal $3.0 \mu \mathrm{M}$; decadienal $1.6 \mu \mathrm{M}+$ octadienal $4.5 \mu \mathrm{M}$; heptadienal $3.0 \mu \mathrm{M}+$ octadienal $4.5 \mu \mathrm{M}$ ) we observed $100 \%$ fertilization success, $100 \%$ first mitotic division after $1 \mathrm{hpf}$ but after $48 \mathrm{hpf}$ all embryos were apoptotic (Supplementary Fig. S1). With the ternary mixtures (decadienal $1.6 \mu \mathrm{M}+$ heptadienal $3.0 \mu \mathrm{M}+$ octadienal $4.5 \mu \mathrm{M}$ ) we also obtained $100 \%$ fertilization success, but only about $50 \%$ of fertilized eggs reached first cleavage and those that did had abnormal two blastomeres (Supplementary Fig. S2). As in the case of binary mixtures all embryos were apoptotic after $48 \mathrm{hpf}$.

In the second experiment, we halved the concentration of the three aldehydes $(0.8 \mu \mathrm{M}$ decadienal, $1.5 \mu \mathrm{M}$ heptadienal and $2.3 \mu \mathrm{M}$ octadienal). In this case the results were the same both for the binary and ternary PUA mixtures with regards to fertilization success (100\%) first mitotic division (100\%) and apoptotic embryos after 48 hpf $(100 \%)$, with the only exception being the binary mixture heptadienal $1.5 \mu \mathrm{M}+$ octadienal $2.3 \mu \mathrm{M}$. In this case the embryos observed at $48 \mathrm{hpf}$ showed a developmental delay and were still at the early pluteus stage compared to control (without PUA mixture) embryos that had reached the late pluteus stage (Supplementary Fig. S3). We followed embryonic development of the early plutei until 1 week post fertilization (wpf) to follow their fate. These embryos reached the pluteus stage even if they were malformed (with degenerate and malformed arms, as pluteus in Supplementary Fig. S3a), that showed delayed development compared to controls (Supplementary Fig. S4), where the embryos showed the characteristic "ampoule-like" shape $e^{31}$.

We then further decreased the three PUA concentrations in the mixtures to one third of the original concentrations, corresponding to $0.5 \mu \mathrm{M}$ decadienal, $1.0 \mu \mathrm{M}$ heptadienal and $1.5 \mu \mathrm{M}$ octadienal. At these concentrations samples incubated with both binary and ternary mixtures showed $100 \%$ fertilization success, $100 \%$ division to two blastomeres at $1 \mathrm{hpf}$. At $48 \mathrm{hpf}$ we observed a very high percentage of embryos at the pluteus stage in all the mixtures tested. More specifically, treatment of eggs with binary and ternary mixtures at 48 hpf produced a significant increase in the number of malformed embryos $(\mathrm{p}<0.0001)$ with respect to the control (Fig. 1b-d). However, only with binary and ternary mixtures containing decadienal we also observed a low percentage of delayed embryos still at the gastrula stage (Fig. 1e).

Gene response to PUA mixtures. Sea urchin eggs were incubated with ternary mixtures of PUAs at concentrations that induced morphological changes but still allowed embryos to develop to the pluteus stage: $0.5 \mu \mathrm{M}$ decadienal, $1.0 \mu \mathrm{M}$ heptadienal and $1.5 \mu \mathrm{M}$ octadienal. To identify the expression profile triggered by PUAs exposure, the expression levels of fifty genes were followed by Real Time qPCR, having key roles in embryonic development, cell differentiation and morphogenesis (see Supplementary Table S1). Their relative expression ratios were calculated respect to control embryos. We considered expression levels greater than two-fold with respect to the controls as significant (see Fig. 2 and Supplementary Table S2).

Stress genes. At the early blastula stage $(5 \mathrm{hpf})$ the gene $c y t b$ were up-regulated $(3.4$-fold, $\mathrm{p}=0.012)$ with respect to the control. At the gastrula stage $(21 \mathrm{hpf})$ seven genes were targeted by the ternary PUA mixture: $h s p 60$ (2.4-, $\mathrm{p}=0.048)$, MTase (2.0, $\mathrm{p}=0.049), p 53$ (2.1, $\mathrm{p}=0.049)$ and ERCC3 (2.8-, $\mathrm{p}=0.035)$ were up-regulated, whereas $h s p 70(2.3-, \mathrm{p}=0.045), 14-3-3 \varepsilon(2.3-, \mathrm{p}=0.045)$ and $N F-k B(2.4-, \mathrm{p}=0.041)$ were down-regulated with respect to the control. At the pluteus stage (48 hpf) nine genes were down-regulated, $h s p 56(2.5-, \mathrm{p}=0.045), G S$ (2.5-, $\mathrm{p}=0.041)$, cytb (7.5, $\mathrm{p}=0.0015)$, p38MAPK (2.3-, $\mathrm{p}=0.039), 14-3-3 \varepsilon(2.7-, \mathrm{p}=0.031)$, caspase 3/7 (2.7-, $\mathrm{p}=0.039)$, caspase- $8(4.0-, \mathrm{p}=0.025), p 53(2.6, \mathrm{p}=0.030)$ e HIF1A $(2.7-, \mathrm{p}=0.031)$. 


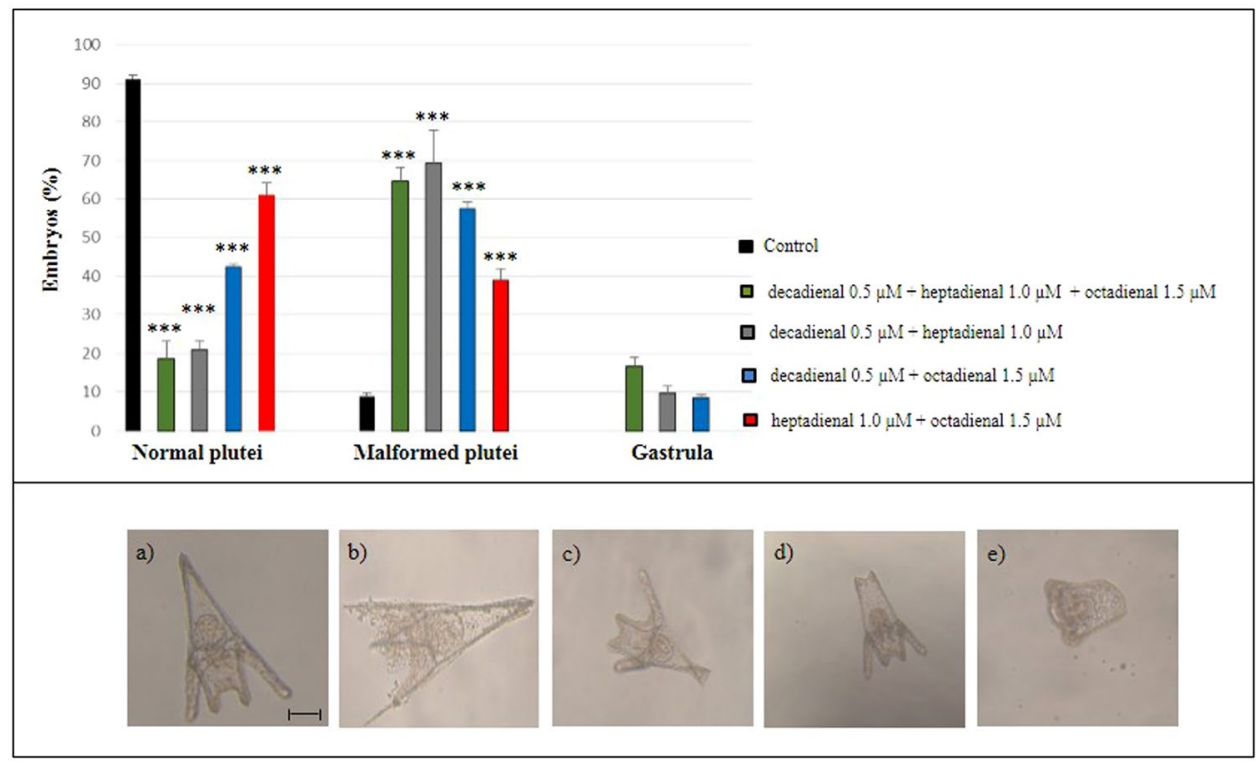

Figure 1. Examples of malformations induced in (b-d) P. lividus plutei at $48 \mathrm{hpf}$ after incubation with binary (decadienal $0.5 \mu \mathrm{M}+$ heptadienal $1.0 \mu \mathrm{M}$; decadienal $0.5 \mu \mathrm{M}+$ octadienal $1.5 \mu \mathrm{M}$; heptadienal $1.0 \mu \mathrm{M}+$ octadienal $1.5 \mu \mathrm{M}$ ) and ternary (decadienal $0.5 \mu \mathrm{M}+$ heptadienal $1.0 \mu \mathrm{M}+$ octadienal $1.5 \mu \mathrm{M}$ ) mixtures in comparison with (a) the control (embryos in sea water without PUA mixtures); (e) embryos still at the gastrula stage. Bar, $50 \mu \mathrm{m}$.

Skeletogenic genes. At the early blastula stage (5 hpf) only the gene BMP5-7 was down-regulated (2.7-fold; $\mathrm{p}=0.011)$ by the PUA mixture. At the gastrula stage $(21 \mathrm{hpf}) S M 30$ and uni were down-regulated (4.3- and 2.0 -fold with $p=0.019$ and $p=0.042$, respectively) whereas BMP5-7, Nec and $p 19$ were up-regulated (5.3-, 4.3and 3.0-fold with $\mathrm{p}=0.0011,0.023$ and 0.029 , respectively). At the pluteus stage (48 hpf) SM30 was up-regulated (2.1-fold, $\mathrm{p}=0.010)$, whereas Nec, p16, p19 and Jun were down-regulated (2.8-, 2.5-, 3.6- and 2.5-fold with $\mathrm{p}=0.011, \mathrm{p}=0.039, \mathrm{p}=0.0023, \mathrm{p}=0.027)$ with respect to the control.

Genes involved in development/differentiation. At the early blastula stage ( $5 \mathrm{hpf})$ all the targeted genes were down-regulated: hat (8.8-, $\mathrm{p}=0.0054), \delta$-2-catenin $(2.4-, \mathrm{p}=0.015), \operatorname{VEGF}(3.1-, \mathrm{p}=0.010)$ and JNK $(3.1$-, $\mathrm{p}=0.0012$ ).

At the gastrula stage $(21 \mathrm{hpf})$ hat and TAK1were down-regulated $(2.2$ - and 2.1 -fold, with $\mathrm{p}=0.028$ and $\mathrm{p}=0.033$ respectively). At the pluteus stage $(48 \mathrm{hpf})$ hat gene was strongly up-regulated $(6.0$-fold, $\mathrm{p}=0.0045)$. On the contrary, fourteen genes were down-regulated: $\operatorname{sox} 9(2.7-, \mathrm{p}=0.019), B P 10(2.6-, \mathrm{p}=0.034)$, Alix (7.6-, $\mathrm{p}<0.0001)$, Wht5 (2.1-, $\mathrm{p}=0.013)$, Wnt8 (3.5-), $\delta$-2-catenin (6.5-, $\mathrm{p}=0.0028)$, nodal (2.7-, $\mathrm{p}=0.021)$, tcf4 (5.1-, $\mathrm{p}=0.0017)$, TCF7 (2.6-, $\mathrm{p}=0.031)$, GFI1 (4.5-, $\mathrm{p}=0.0045)$, Onecut/Hnf6 (3.4-, $\mathrm{p}=0.01)$, TAK1 (5.2-, $\mathrm{p}=0.0025)$, $\operatorname{VEGF}(2.3-, \mathrm{p}=0.039) J N K(2.0-, \mathrm{p}=0.045)$.

Genes involved in detoxification. At the early blastula stage (5 hpf) only MT gene was down-regulated (3.4-fold, $\mathrm{p}=0.018)$. At the gastrula stage $(21 \mathrm{hpf}) M T$ and $C A T$ were up-regulated (3.9- and 2.0 -fold with $\mathrm{p}=0.016$ and $\mathrm{p}=0.043$, respectively). At the pluteus stage ( $48 \mathrm{hpf}$ ) MT4 was up-regulated (3.3-fold, $\mathrm{p}=0.021$ ), whereas MT5, $M T 7, M T 8$ e MDR1 were down-regulated (3.2-, 7.0-, 2.9- and 2.9-fold with $\mathrm{p}=0.011, \mathrm{p}<0.0009, \mathrm{p}=0.023$, $\mathrm{p}=0.019$, respectively).

Statistically significant differences were found between gene expression values at $48 \mathrm{hpf}$ versus both $5 \mathrm{hpf}$ $(\mathrm{p}<0.001)$ and $21 \mathrm{hpf}(\mathrm{p}<0.0001)$.

Summarizing, PUA mixtures were able to switch on the expression levels of almost all analyzed genes, with the exception of SM50, Blimp, Wnt6, FoxG, FOXA, Foxo and MT6 (for a synopsis see Supplementary Fig. S7). All gene functional classes were affected by PUA mixtures but at different developmental stages. At the blastula stage only seven genes were targeted by PUA mixtures in the four functional classes, probably since it is too early to detect an effect on gene expression at this stage. At the gastrula stage, the number of affected genes increased, with sixteen genes varying in their gene expression: stress genes (seven genes) and those involved in skeletogenesis (seven genes) were mainly affected, indicating that embryos started to react against these toxic aldehydes. At the pluteus stage, when the entire body was well-formed, thirty four genes were affected by the PUA mixture, demonstrating that the embryos were trying to defend themselves by placing in motion a greater number of genes. In particular, the PUA mixture mainly affected genes involved in stress (nine genes) and in development and differentiation processes (fifteen genes). It is important to underline that some previous results demonstrated that most of these genes were intercorrelated ${ }^{28,30}$. 


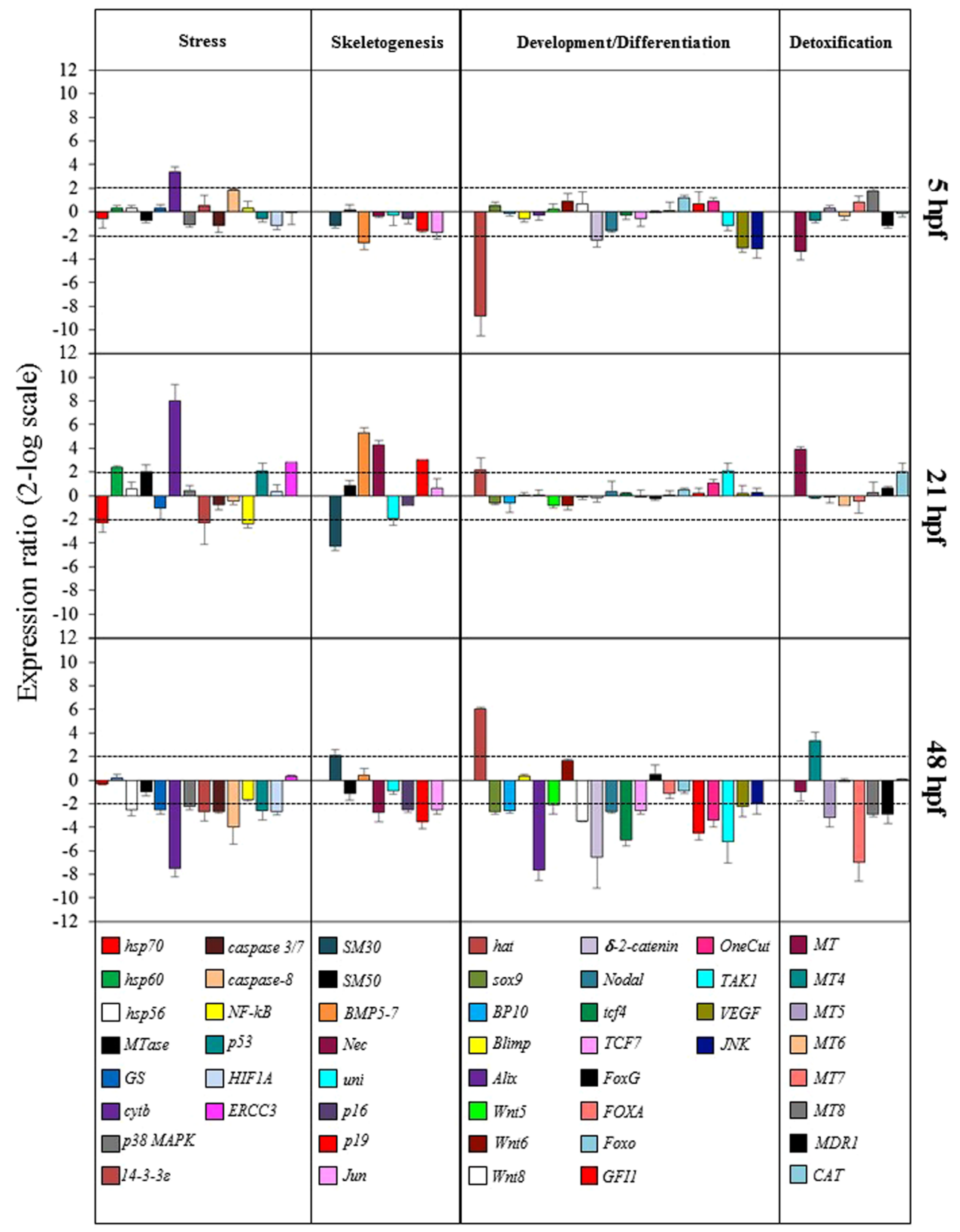

Figure 2. Real-Time qPCR at blastula (5hpf), gastrula ( $21 \mathrm{hpf}$ ) and pluteus ( $48 \mathrm{hpf})$ stages. Histograms show the differences in expression levels of fifty genes involved in different embryonic processes, divided in four classes: stress, skeletogenesis, development/differentiation and detoxification. P. lividus embryos were grown in the presence of ternary PUA mixture, consisting of decadienal $0.5 \mu \mathrm{M}$, heptadienal $1.0 \mu \mathrm{M}$ and octadienal $1.5 \mu \mathrm{M}$. Data are reported as a fold difference compared with control (mean $\pm \mathrm{SD}$ ) embryos in sea water without PUA mixture. Fold differences greater than \pm 2 (see red dotted horizontal guidelines at values of +2 and -2 ) were considered significant (see Supplementary Table S2 for the values).

Heat maps of differentially expressed genes. We have constructed three heatmaps, one for each developmental stage, considering the three females for both control and after PUAs exposure (see Supplementary Fig. S5). Real-Time $q$ PCR data were presented as individual data points as $2^{-\Delta C}{ }_{T}^{39}$. Our results showed that the three females in the control conditions (in seawater without PUAs, indicated with C1, C2 and C3) and after treatment with PUA mixture (indicated with T1, T2 and T3) clustered in two separate groups within the dendrogram. Moreover, differences within the same groups (within the control samples and treated samples) were also evident. These results reflected a fraction of the variability that is likely to be expected in this species.

A heat map of differentially expressed genes versus developmental stages is reported in Fig. 3, which shows the high level of gene expression variability among the three different developmental stages considered, increasing from the blastula stage to pluteus stage where a down-regulation of genes was very evident. 


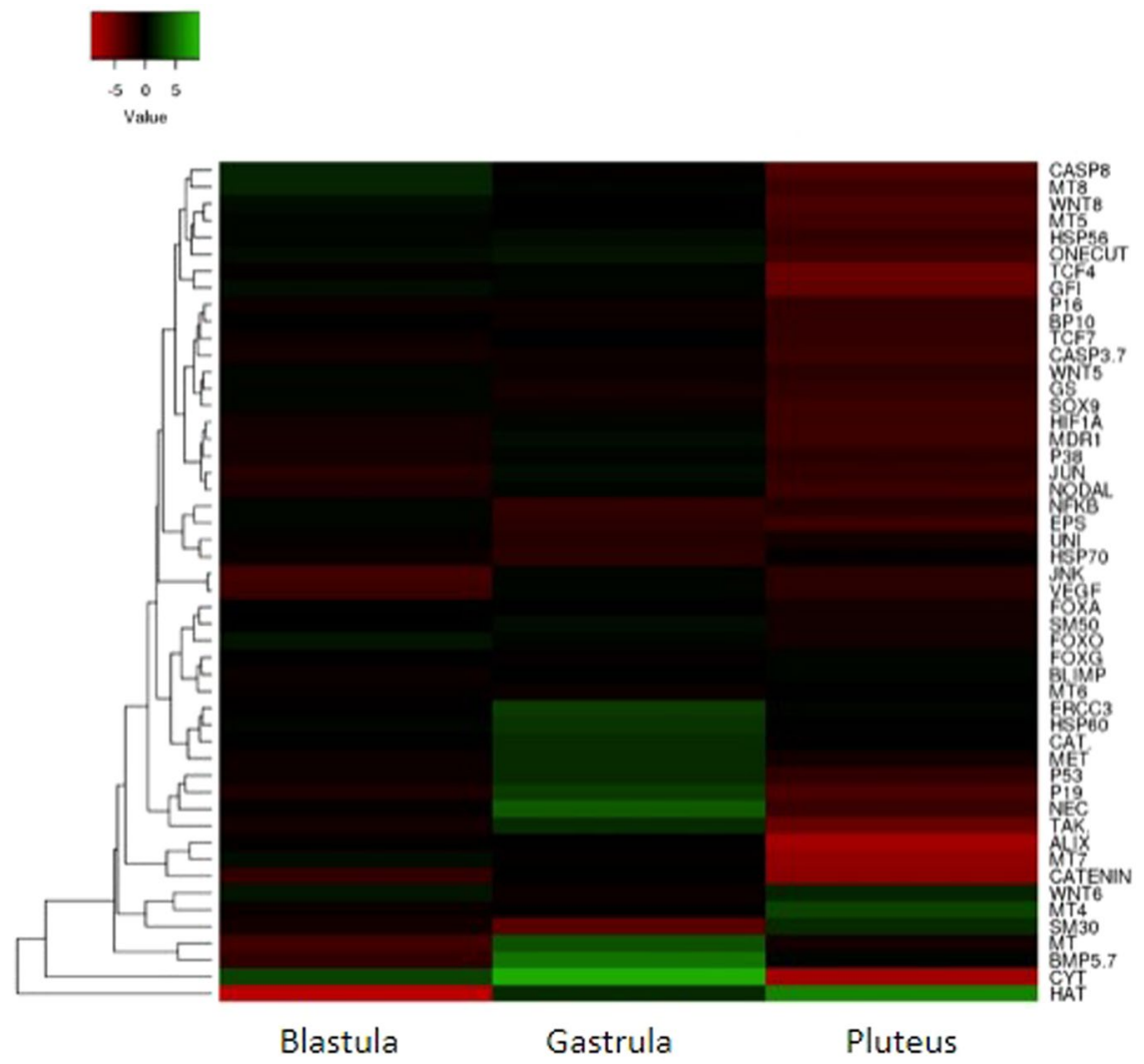

Figure 3. Heat map (using Heatmapper available at www.heatmappear.ca; https://creativecommons.org/ licenses/by-sa/2.0/) of differentially expressed genes versus the three developmental stages (early blastula, late gastrula and pluteus), at which developing sea urchin P. lividus embryos have been collected after PUA mixture at $0.5 \mu \mathrm{M}$ decadienal, $1.0 \mu \mathrm{M}$ heptadienal and $1.5 \mu \mathrm{M}$ octadienal for Real Time qPCR. Color code: red, negative values of gene expression (down-regulated genes respect to the control, embryos developed in sea water without PUA mixture); green, positive values of gene expression (up-regulated genes respect to the control); black, genes for which there was no variation of expression respect to the control.

This high variability at the population level was also evident in the heat map of gene expression versus the three females (indicated with F1, F2 and F3; Supplementary Fig. S6), where F2 seems to differ somewhat in terms of its response in gene expression compared to F1 and F3.

\section{Discussion}

In the present study, the effects of PUA mixtures on early stages of embryonic development in the sea urchin $P$. lividus are evaluated for the first time. These results greatly expand our previous investigations using single PUAs, decadienal, heptadienal and octadienal, to study the stress response induced of the sea urchin. Heptadienal and octadienal are two ecologically important diatom-derived PUAs, but relatively unknown in terms of their effects on grazers, in comparison with the extensively studied PUA, decadienal ${ }^{24-30,40,41}$. Our results also provide information on the transmission of "maternal" stress induced by PUAs to their progeny at the morphological and molecular levels. In sea urchin eggs maternal mRNAs drive the first mitotic cell cycles starting from fertilization to the onset of zygotic transcription, independently of mRNA transcription and ribosome biogenesis ${ }^{42-44}$.

Several ecotoxicological studies concern single compound exposures, although the toxicity of certain compounds increases in mixtures and in presence of other pollutants. Our findings take an alternative look at a relatively well-studied area of marine chemical ecology, making significant advances on PUA effects in mixtures and not limited to single PUA exposures.

To date, all the published data have reported the negative effects of individual PUAs on marine invertebrates. There are some studies reporting the effect of decadienal, as representative of PUAs, in the presence of sub-lethal levels of heavy metals on the rotifer Brachionus plicatilis and nauplii of the brine shrimp Artemia salina ${ }^{45}$. Also in this case, decadienal was the most toxic to both species. The presence of $1 \mu \mathrm{M}$ of copper sulphate and zinc sulphate in solutions of decadienal resulted in an 11 and 3\% reduction in the LD50 (Lethal Dose 50\%, the concentration required to kill $50 \%$ of the test population) of decadienal compared to the $33 \%$ reduction with $1 \mu \mathrm{M}$ copper sulphate.

Here, we investigated the effects induced not by individual PUAs, but by PUA mixtures. More specifically, we tested binary and ternary mixtures, demonstrating that PUAs in mixtures acted in a synergistic way, because their effect was stronger than individual PUAs, with negative impacts on sea urchin embryos. When we used binary and ternary mixtures at the concentrations that produced about $35 \%$ of malformed embryos with individual 
PUAs (decadienal 1.6 $\mu \mathrm{M}$, heptadienal 3.0 $\mu \mathrm{M}$ and octadienal $4.5 \mu \mathrm{M})^{29}$, all the embryos were apoptotic. Only at PUA concentrations corresponding to one third of these concentrations (corresponding to decadienal $0.5 \mu \mathrm{M}$, heptadienal $1.0 \mu \mathrm{M}$ and octadienal $1.5 \mu \mathrm{M}$ ), were embryos able to develop to the pluteus stage. According to Varrella et al. ${ }^{29}$ at these concentrations, individual PUAs have no effects on sea urchin embryos with values similar to the control (embryos in filtered sea water without PUAs). Moreover, our results confirm that decadienal was the strongest PUA, because all the PUA mixtures tested containing decadienal always had stronger toxicity for $P$. lividus embryos. These results corroborate previous data reporting that decadienal affected sea urchin embryonic development in a very narrow range $(2.0 \mu \mathrm{M})$; whereas heptadienal and octadienal required higher ranges of concentrations to reach the same effects as decadienal ${ }^{29}$.

The stronger effects of PUA mixtures in comparison with individual PUAs observed at the morphological level, was also confirmed by molecular results. The expression levels of several genes in the sea urchin P. lividus, appeared to be modulated by individual PUAs decadienal, heptadienal and octadienal. These three aldehydes had some common targets ( 15 out of 50 genes analyzed), differently affecting the classes of genes at different embryonic stages ${ }^{29}$. In fact, heptadienal induces the strongest molecular effects, affecting the expression levels of thirteen genesbut at the pluteus stage ${ }^{30}$. Even if octadienal results the weakest of the three aldehydes by morphological point of view, our previous study on gene expression levels revealed that it affects early embryonic development. Decadienal seems to be molecularly the weakest of the three PUAs, but it has specific molecular targets. Therefore, although no morphologically differences are visible, PUAs affected different physiological processes.

Combining standard embryological assays with gene expression is useful to tease out interesting data in terms of how the PUA mixtures interact with embryo transcriptome. The present study highlights substantial gene plasticity in embryos of the sea urchin P. lividus in response to PUA mixtures. More specifically, embryos subjected to the PUA mixture reduced and delayed response at the transcript level, taking into account that the greatest number of genes was down-regulated and mainly at the pluteus stage. These data suggest that the physiological response in the sea urchin P. lividus may be impaired at the molecular level after PUAs mixture exposure. These data are in accordance with some experiments performed on the larvae from the red sea urchin Strongylocentrotus franciscanus, showing a reduced and delayed response in $h s p 70$ transcript level when exposed to elevated $\mathrm{CO}_{2}$ conditions and then subjected to a temperature shift ${ }^{46}$. In that case, the Authors suggested that marine organisms could protect themselves against ocean acidification, costing a lot for the organism's ability to tolerate increased stress. Molecular plasticity has been previously demonstrated also in a mollusc population, when subjected to environmental stress ${ }^{47,48}$. In this case larger-scale genomic studies have been performed, appearing as one of the most rapid and sensitive way to study the responses to environmental stressors. Martin et al. ${ }^{49}$ focused their attention in understanding if ocean acidification affect the initial developmental stages of $P$. lividus. In fact, this organisms appeared extremely resistant to low $\mathrm{pH}$, showing no effect on fertilization success or larval survival, but only delay of the larval development. The analyzed genes, involved in developmental and biomineralization processes, were surprisingly upregulated at low $\mathrm{pH}$, so revealing that plasticity at the gene expression level was able to produce a normal, but delayed embryonic development under low $\mathrm{pH}$ conditions.

Finally, it would be interesting to consider PUA ratios, but to our knowledge, the only data available are those for Skeletonema marinoi, which does not produce decadienal but only heptadienal and octadienal. Vidoudez and Pohnert ${ }^{50}$ reported that heptadienal was at least 3 times more abundant than octadienal. Both PUAs were mainly produced in the late stationary phase when a dramatic increase in heptadienal and octadienal was observed, with concentrations reaching $290 \mathrm{nM}$ heptadienal and $86 \mathrm{nM}$ octadienal. These two PUAs were almost entirely absent in the other growth phases. There is also limited information for another diatom species, Thalassiosira rotula, which produces all three PUAs, but the only study available provides information on the total quantity of PUAs and not for each of the three individual aldehydes ${ }^{51}$.

\section{Conclusions}

To our knowledges, this work represents the first report on the effects of diatom-derived PUAs mixtures on the sea urchins. Our findings reveal a synergistic toxic effect of diatom-derived aldehyde mixtures on embryonic $P$. lividus development, also confirming that the sea urchin embryos can be considered a good model to study the stress defence mechanisms in marine invertebrates. Finally, this represents an important ecological finding, considering that marine organisms are exposed to diatom-derived oxylipin mixtures, offering new knowledges on how marine organisms try to react and to defend themselves against environmental natural toxin mixtures such as diatom-derived PUAs.

\section{Materials and Methods}

Ethics Statement. Paracentrotus lividus (Lamarck) adults were collected in the Bay of Naples according to the Italian legislation (DPR 1639/68, 09/19/1980 confirmed on 01/10/2000). Field studies did not include endangered or protected species. All experimental procedures on animals were in compliance with the guidelines of the European Union (Directive 609/86).

Morphological experiments. Concerning the collection of adult sea urchin P. lividus and the procedure for gametes collection see Varrella et al. ${ }^{29,31}$. Eggs were treated for 10 minutes with binary and ternary mixtures of 2-trans,4-trans-decadienal (Sigma-Aldrich), 2-trans,4-trans-heptadienal (Sigma-Aldrich), and 2-trans,4-trans-octadienal (Sigma-Aldrich) at the following concentrations:

1. 2-trans,4-trans-decadienal $1.6 \mu \mathrm{M}, 2$-trans,4-trans-heptadienal $3.0 \mu \mathrm{M}, 2$-trans, 4 -trans-octadienal a $4.5 \mu \mathrm{M}$;

2. 2-trans,4-trans-decadienal $0.8 \mu \mathrm{M}, 2$-trans,4-trans-heptadienal $1.5 \mu \mathrm{M}, 2$-trans,4-trans-octadienal $2.3 \mu \mathrm{M}$;

3. 2-trans,4-trans-decadienal $0.5 \mu \mathrm{M}, 2$-trans,4-trans-heptadienal $1.0 \mu \mathrm{M}, 2$-trans,4-trans-octadienal $1.5 \mu \mathrm{M}$; 
Eggs were then fertilized. Experiments were conducted in triplicates for each group of concentrations, using eggs from ten different females. Control experiment consists in fertilizing eggs in filtered sea water without PUA mixtures. The development was followed until 48 hours post fertilization (hpf) and then embryos were fixed with formaldehyde (4\% in FSW) in treated and control samples for microscopic observation, in order to detect the percentage of abnormal plutei, (Zeiss Axiovert 135TV, Carl Zeiss, Jena, Germany): control samples (in FSW without PUAs) showed about $90 \%$ of normal plutei and about $10 \%$ of malformed embryos.

Differences between normal and malformed plutei and embryos still at the gastrula stage exposed to different PUA mixtures were evaluated as mean \pm standard deviation (SD) and Student's $t$ tests were performed with Prism 3.0 software (GraphPad Prism 4.00 for Windows, GraphPad Software, San Diego California USA). P $<0.05$ was considered as statistically significant.

Molecular experiments. Concerning the collection of eggs and total RNA extraction using RNAqueous ${ }^{\circledR}$ Micro Kit (Ambion from Life Technologies), see Ruocco et al. ${ }^{52}$. The expression level of 50 genes previously analyzed genes were followed by Real-Time $q P C R^{24-31}$ (Supplementary Table S1). The expression of each gene was analyzed and internally normalized against $\mathrm{Pl}-\mathrm{Z12}$-1 (a zinc-finger transcription factor $)^{53}$ using REST software (Relative Expression Software Tool, Weihenstephan, Germany) based on the Pfaffl method ${ }^{54,55}$. PCR efficiencies were verified by melting curve analysis ${ }^{24,28,30}$. One microliter of cDNA was used as a template. For further details on quantification and qualitative analysis of RNA see Ruocco et al. ${ }^{52}$ ). Relative expression ratios above two cycles were considered significant. For each Real Time qPCR plate the experiments were repeated at least twice. We checked that all genes whose fold-change in expression was $> \pm 2$ compared to the control were statistically significant. A Mantel test was applied.

Heat map of the differentially expressed genes versus developmental times for three different females were constructed using Heatmapper ${ }^{56}$ available at www.heatmappear.ca. A hierarchical clustering was performed through the Euclidean distance measurement method and an average linkage clustering method.

When real-time PCR data are presented as individual data points they should be presented as $2^{-\Delta C_{T}}$ or $2^{-C_{T}}$ rather than the raw $\mathrm{C}_{\mathrm{T}}$ value ${ }^{39}$. In this way, the data from gene expression profiling studies are normalize to an internal control. Heatmaps generally undergo a log transformation.

Concerning the morphological data to evidence differences between mixtures for both normal and malformed groups, statistical analysis was performed using GraphPad Prism version 4.00 for Windows (GraphPad Software, San Diego, CA, USA). Data of expression level did not pass the D’Agostino and Person normality test, indicating that One-way ANOVA was not applicable to the data sets. However, a non-parametric Kruskal-Wallis test was applied, followed by Dunn's post-hoc test.

\section{References}

1. Kozlowsky-Suzuki, B., Koski, M., Hallberg, E., Wallén, R. \& Carlsson, P. Glutathione transferase activity and oocyte development in copepods exposed to toxic phytoplankton. Harmful Algae. 8, 395-406 (2009).

2. Altenburger, R., Scholz, S., Schmitt-Jansen, M., Busch, W. \& Eschert, B. I. Mixture toxicity revisited from a toxicogenomic perspective. Environ. Sci. Technol. 46, 2508-2522 (2012).

3. Kortenkamp, A. Ten years of mixing cocktails: A review of combination effects of endocrine-disrupting chemicals. Environ. Health Perspect. 115, 98-105 (2007).

4. Mumatz, M. M., Hugh, H. \& Pohl, H. R. Mixtures and their risk assessment in toxicology. Met. Ions Life Sci. 8, 61-80 (2011).

5. Teuschler, L. et al. Support of science-based decisions concerning the evaluation of the toxicology of mixtures: a new beginning. Regul. Toxicol. Pharmacol. 36, 34-39 (2002).

6. Yang, R. S. H. et al. Chemical mixture toxicology: from descriptive to mechanistic and going on to in silico toxicology. Environ. Toxicol. Pharmacol. 18, 65-81 (2004).

7. Koppe, J. G. et al. Exposure to multiple environmental agents and their effect. Acta Paediatr. Suppl. 95, 106-113 (2006).

8. Backhaus, T. et al. Proposal for environmental mixture risk assessment in the context of the biocidal product authorization in the EU. Environ. Sci. Eur. 25, 4 (2013).

9. Xie, F. et al. Assessment of the toxicity of mixtures of copper, 9,10-phenanthrenequinone, and phenanthrene to Daphnia magna: Evidence for a reactive oxygen mechanism. Environ. Toxicol. Chem. 25, 613-622 (2006).

10. Cutignano, A. et al. Chloroplastic glycolipids fuel aldehyde biosynthesis in the marine diatom Thalassiosira rotula. Chem Bio Chem. 7, 450-456 (2006).

11. d'Ippolito, G. et al. The role of complex lipids in the synthesis of bioactive aldehydes of the marine diatom Skeletonema costatum. Biochim. Biophys. Acta. 1686, 100-107 (2004).

12. Fontana, A. et al. LOX-induced lipid peroxidation mechanism responsible for the detrimental effect of marine diatoms on zooplankton grazers. ChemBioChem. 8, 1810-1818 (2007).

13. Pohnert, G. Wound-activated chemical defence in unicellular planktonic algae. Angew. Chem. Int. Ed. 39, 4352-4354 (2000).

14. Pohnert, G. Diatom-Copepod interactions in plankton: the indirect chemical defense of unicellular algae. Chem. Biochem. 6, 1-14 (2005).

15. Ribalet, F. et al. Phytoplankton cell lysis associated with polyunsaturated aldehyde release in the Northern Adriatic Sea. PLoS ONE. 9, e85947 (2014)

16. Miralto, A. et al. The insidious effect of diatoms on copepod reproduction. Nature. 402, 173-176 (1999).

17. Caldwell, G. S., Olive, P. J. W. \& Bentley, M. G. Inhibition of embryonic development and fertilization in broadcast spawning marine invertebrates by water soluble diatom extracts and the diatom toxin 2-trans,4-trans decadienal. Aquat. Toxicol. 60, 123-137 (2002).

18. Caldwell, G. S., Bentley, M. G. \& Olive, P. J. W. First evidence of sperm motility inhibition by the diatom aldehyde 2 E,4E-decadienal. Mar. Ecol. Progr. Ser. 273, 97-108 (2004).

19. Caldwell, G. S. The influence of bioactive oxylipins from marine diatoms on invertebrate reproduction and development. Mar. Drugs. 7, 367-400 (2009)

20. Lettieri, A., Esposito, R., Ianora, A. \& Spagnuolo, A. Ciona intestinalis as a marine model system to study some key developmental genes targeted by the diatom-derived aldehyde decadienal. Mar. Drugs. 13, 1451-1465 (2015).

21. Tosti, E. et al. Bioactive aldehydes from diatoms block fertilization currents in ascidian oocytes. Mol. Rep. Dev. 66, 72-80 (2003).

22. Ianora, A. \& Miralto, A. Toxigenic effects of diatoms on grazers, phytoplankton and other microbes: a review. Ecotoxicology. 19, 493-511 (2010).

23. Hansen, E., Even, Y. \& Geneviere, A. M. The alpha, beta, gamma, deltaunsaturated aldehyde 2-trans-4-trans-decadienal disturbs DNA replication and mitotic events in early sea urchin embryos. Toxicol. Sci. 81, 190-197 (2004). 
24. Marrone, V. et al. Defensome against toxic diatom aldehydes in the sea urchin Paracentrotus lividus. PLoS ONE. 7, e31750 (2012).

25. Romano, G., Miralto, A. \& Ianora, A. Teratogenic effects of diatom metabolites on sea urchin Paracentrotus lividus embryos. Mar. Drugs. 8, 950-967 (2010).

26. Romano, G., Costantini, M., Buttino, I., Ianora, A. \& Palumbo, A. Nitric oxide mediates the stress response induced by diatom aldehydes in the sea urchin Paracentrotus lividus. PloS ONE. 6, e25980 (2011).

27. Ruocco, N. et al. Diatom-derived oxylipins induce cell death in sea urchin embryos activating caspase-8 and caspase 3/7. Aquat. Toxicol. 176, 128-140 (2016).

28. Ruocco, N. et al. New inter-correlated genes targeted by diatom-derived polyunsaturated aldehydes in the sea urchin Paracentrotus lividus. Ecotoxicol. Environ. Saf. 142, 355-362 (2017b).

29. Varrella, S. et al. Molecular response to toxic diatom-derived aldehydes in the sea urchin Paracentrotus lividus. Mar. Drugs. 12, 2089-2113 (2014)

30. Varrella, S. et al. Toxic diatom aldehydes affect defence gene networks in sea urchins. PloS ONE. 11, e0149734 (2016a).

31. Varrella, S. et al. First morphological and molecular evidence of the negative impact of diatom-derived hydroxyacids on the sea urchin Paracentrotus lividus. Toxicol. Sci. 151, 419-433 (2016b).

32. Sansone, C. et al. Diatom-derived polyunsaturated aldehydes activate cell death in human cancer cell lines but not normal cells. PLoS ONE. 9, e101220 (2014)

33. Ribalet, F., Berges, J. A., Ianora, A. \& Casotti, R. Growth inhibition of cultured marine phytoplankton by algal-derived polyunsaturated aldehydes. Aquat. Toxicol. 85, 219-227 (2007a).

34. Pinsino, A. \& Matranga, V. Sea urchin immune cells as sentinels of environmental stress. Dev. Comp. Immunol. 49, 198-205 (2015).

35. Roepke, T. A., Snyder, M. J. \& Cherr, G. N. Estradiol and endocrine disrupting compounds adversely affect development of sea urchin embryos at environmentally relevant concentrations. Aquat. Toxicol. 71, 155-173 (2005).

36. Todgham, A. E. \& Hofmann, G. E. Transcriptomic response of sea urchin larvae Strongylocentrotus purpuratus to $\mathrm{CO}_{2}$-driven seawater acidification. J. Exp. Biol. 212, 2579-2594 (2009).

37. Strathmann, M. F. Reproduction and development of marine invertebrates of the Northern Pacific Coast. University of Washington Press, Seattle, WA, USA, pp. 670 (1987).

38. Sodergren, E. et al. The genome of the sea urchin Strongylocentrotus purpuratus. Science. 314, 941-952 (2006).

39. Schmittgen, T. D. \& Livak, K. J. Analyzing real-time PCR data by the comparative $\mathrm{C}_{\mathrm{T}}$ method. Nature Protocols. 3, 1101-1108 (2008).

40. Wichard, T. et al. Survey of the chemical defence potential of diatoms: screening of fifty species for $\alpha, \beta, \gamma, \delta$-unsaturated aldehydes. $J$. Chem. Ecol. 31, 949-958 (2005).

41. Wichard, T. et al. Lipid and fatty acid composition of diatoms revisited: rapid wound-activated change of food quality parameters influences herbivorous copepod reproductive success. ChemBioChem. 8, 1146-1153 (2007).

42. Davidson, E. H. The nature and function of maternal transcripts. In: Gene activity in early development. Academic Press, New York, USA, pp. 46-125 (1986).

43. Tadros, W. \& Lipshitz, H. D. The maternal-to-zygotic transition: a play in two acts. Development. 136, 3033-3042 (2009).

44. Runcie, D. E. et al. Genetics of gene expression responses to temperature stress in a sea urchin gene network. Mol. Ecol. 21, 4547-4562 (2012).

45. Taylor, R. L., Caldwell, G. S. \& Bentley, M. G. Toxicity of algal derived aldehydes to two invertebrate species: Do heavy metal pollutants have a synergistic effect? Aquat. Toxicol. 74, 20-31 (2005).

46. O’Donnell, M. J. et al. Ocean acidification alters skeletogenesis and gene expression in larval sea urchins. Mar. Ecol. Prog. Ser. 398, 157-171 (2010).

47. Gracey, A. Y. et al. Rhythms of gene expression in a fluctuating intertidal environment. Curr. Biol. 18, 1501-1507 (2008).

48. Place, S. P., O’Donnell, M. J. \& Hofmann, G. E. Gene expression in the intertidal mussel Mytilus californianus: physiological response to environmental factors on a biogeographic scale. Mar. Ecol. Prog. Ser. 356, 1-14 (2008).

49. Martin, S. et al. Early development and molecular plasticity in the Mediterranean sea urchin Paracentrotus lividus exposed to $\mathrm{CO}_{2-}$ driven acidification. J. Exp. Biol. 214, 1357-1368 (2011).

50. Vidoudez, C. \& Pohnert, G. Growth phase-specific release of polyunsaturated aldehydes by the diatom Skeletonema marinoi. J. Plankton Res. 30, 1305-1313 (2008).

51. Barreiro, A. et al. Diatom induction of reproductive failure in copepods: The effect of PUAs versus non volatile oxylipins. J. Exp. Mar. Biol. Ecol. 401, 13-19 (2011).

52. Ruocco, N. et al. High-quality RNA extraction from the sea urchin Paracentrotus lividus embryos. PLoS ONE. 12, e0172171 (2017a).

53. Costa, C., Karakostis, K., Zito, F. \& Matranga, V. Phylogenetic analysis and expression patterns of p16 and p19 in Paracentrotus lividus embryos. Dev. Genes Evol. 222, 245-251 (2012).

54. Pfaffl, M. W. A new mathematical model for relative quantification in realtime RT-PCR. Nucleic Acid Res. 29, e45 (2001).

55. Pfaffl, M. W., Horgan, G. W. \& Dempfle, L. Relative expression software tool (REST) for group-wise comparison and statistical analysis of relative expression results in real-time PCR. Nucleic Acid Res. 30, e36 (2002).

56. Babicki, S. et al. Heatmapper: web-enabled heat mapping for all. Nucleic Acids Res. 44, W147-153 (2016).

\section{Acknowledgements}

Nadia Ruocco (Ph.D. in Biology, University of Naples Federico II) has been supported by a Ph.D. fellowship cofounded by the Stazione Zoologica Anton Dohrn and Bio-Organic Chemistry Unit of the Institute of Biomolecular Chemistry-CNR, Pozzuoli, Naples (Italy). We thank the Fishing Service of the SZN for providing sea urchins. Many thanks are also due to Davide Caramiello of the Marine Resources for Research Unit for his technical support in sea urchin maintenance and gamete collection. We also thank Massimo Perna of the Department of Marine Biotechnology for providing formaldehyde to fix embryos.

\section{Author Contributions}

N.R., A.I., G.L., L.M., M.C. designed research; C.A., N.R. performed experiments; S.C. performed bioinformatics analysis; M.C., A.I. contributed new reagents or analytical tools; N.R., A.I., G.L., L.M., S.C., M.C. analyzed data; all the authors contributed to write the paper.

\section{Additional Information}

Supplementary information accompanies this paper at https://doi.org/10.1038/s41598-018-37546-y.

Competing Interests: The authors declare no competing interests.

Publisher's note: Springer Nature remains neutral with regard to jurisdictional claims in published maps and institutional affiliations. 
(i) Open Access This article is licensed under a Creative Commons Attribution 4.0 International License, which permits use, sharing, adaptation, distribution and reproduction in any medium or format, as long as you give appropriate credit to the original author(s) and the source, provide a link to the Creative Commons license, and indicate if changes were made. The images or other third party material in this article are included in the article's Creative Commons license, unless indicated otherwise in a credit line to the material. If material is not included in the article's Creative Commons license and your intended use is not permitted by statutory regulation or exceeds the permitted use, you will need to obtain permission directly from the copyright holder. To view a copy of this license, visit http://creativecommons.org/licenses/by/4.0/.

(C) The Author(s) 2019 